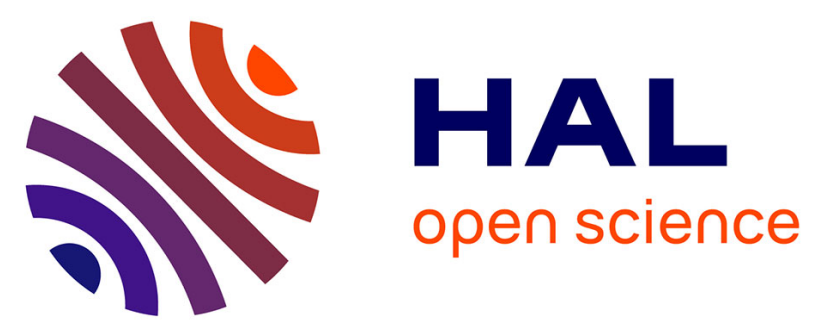

\title{
New characterization methods for monitoring small resonant frequency variation: Experimental tests in the case of hydrogen detection with uncoated silicon microcantilever-based sensors
}

\author{
Mohand-Tayeb Boudjiet, Johan Bertrand, Claude Pellet, Isabelle Dufour
}

\section{To cite this version:}

Mohand-Tayeb Boudjiet, Johan Bertrand, Claude Pellet, Isabelle Dufour. New characterization methods for monitoring small resonant frequency variation: Experimental tests in the case of hydrogen detection with uncoated silicon microcantilever-based sensors. Sensors and Actuators B: Chemical, 2014, 199, pp.269-276. 10.1016/j.snb.2014.03.098 . hal-00964871

\section{HAL Id: hal-00964871 \\ https://hal.science/hal-00964871}

Submitted on 13 Nov 2014

HAL is a multi-disciplinary open access archive for the deposit and dissemination of scientific research documents, whether they are published or not. The documents may come from teaching and research institutions in France or abroad, or from public or private research centers.
L'archive ouverte pluridisciplinaire HAL, est destinée au dépôt et à la diffusion de documents scientifiques de niveau recherche, publiés ou non, émanant des établissements d'enseignement et de recherche français ou étrangers, des laboratoires publics ou privés. 


\title{
New characterization methods for monitoring small resonant frequency variation: Experimental tests in the case of hydrogen detection with uncoated silicon microcantilever-based sensors.
}

\author{
M.T. Boudjiet ${ }^{1}$, J. Bertrand ${ }^{2}$, C. Pellet ${ }^{1}$, I. Dufour ${ }^{1}$ \\ ${ }^{1}$ Univ. Bordeaux, IMS, UMR 5218, F-33400 Talence, France \\ ${ }^{2}$ Andra, F-92298 Châtenay-Malabry, France
}

\begin{abstract}
The uncoated silicon microcantilever (USMC) operated in the dynamic mode is a new concept in the field of microcantilever-based chemical sensors. Due to the absence of a sensitive layer, this kind of microsensor can only be used for specific applications where it is known that only one chemical species may be varying in concentration, such as monitoring hydrogen release in radioactive waste disposal facilities. Usually, the relative variation of the USMC resonant frequency expected for low concentrations $(\leq 2 \%)$ of hydrogen in nitrogen is below 50ppm. As a result, the measurement of both the resonant frequency, $f_{r}$, and the quality factor, $Q$, by classical methods, based on the gain spectrum (resonant peak and $-3 \mathrm{~dB}$ bandwidth), is not sufficiently accurate. In this paper, new measurement methods for monitoring $f_{r}$ and $Q$ variations are proposed: (1) variation of gain and phase at fixed frequencies and (2) polynomial approximations of gain and phase spectra. The performance study of these characterization methods shows that monitoring $f_{r}$ by using phase linearization yields the best signal-to-noise ratio (e.g., 100 at $0.6 \%$ of $\mathrm{H}_{2}$ in $\mathrm{N}_{2}$ ), with $0.02 \%$ as a limit of detection for hydrogen.
\end{abstract}

\section{Keywords}

Resonant frequency, quality factor, uncoated silicon microcantilever, chemical detection, signal-to-noise ratio.

\section{INTRODUCTION}

\subsection{Hydrogen release in radioactive waste disposal facility}

After having concluded a feasibility study on deep geological disposal for high-level and Intermediate level long-lived radioactive waste in 2005, the French national radioactive waste management agency (Andra) was charged by the Planning Act n²006-739 to study the design and the creation of an industrial center for geological disposal called Cigéo which must be reversible for at least a century-long period. Within the framework of this geological repository project, the observation and surveillance must contribute to acquire the knowledge required to run the disposal and its reversible management. Hydrogen release is expected in the radioactive waste disposal facility. It originates from (i) radioactive waste release (ii) and anoxic corrosion of metallic materials. In fact, some radioactive wastes (containing $\alpha, \beta, \gamma$ radioactivity) resulting from the reprocessing of irradiated fuels are embedded in bitumen matrix. The self-irradiation of the bituminized waste leads mainly to the production of radiolytic hydrogen (75-95 vol. $\%$ of produced radiolytic gas). In the deep geological disposal environments steel components would corrode to more oxidized corrosion products and hydrogen gas. The kinetic of Anoxic corrosion of Fe components is not well known, it is actually considered an average rate about $10 \mu \mathrm{m} / \mathrm{year}$. This assumption still awaits experimental verification in the Underground Research Laboratory sited at Bure in the Meuse district and aims at studying the feasibility of the reversible geological disposal of high-level and long-lived intermediate-level radioactive waste in the Callovo-Oxfordian clay formation (east of France). Despite the fact that hydrogen releases are expected to be small (in the order of $430 \mathrm{mmol} /$ hour for each intermediate level nuclear waste), when ventilation stops with cell closure, concentrations would slowly and regularly increase. 
The first calculation gives $4 \%$ hydrogen content in the atmosphere of the radioactive waste cell in less than one year by the end of the oxic corrosion period.

Monitoring of repository structures contributes to security, safety and reversible management of the repository [1]. It is important as it relates to the guidance of the disposal process and to the corresponding decision-making process. In the upstream repositorydesign phases, the hydrogen monitoring system was planned to work under normal operating conditions and to withstand radiation exposure in case of an accidental event. There exist different kinds of hydrogen sensors, including those that are commercially available and others still under development [2]. In the present paper, the preliminary results concerning the development of microcantilever-based sensors without sensitive coating (Uncoated Silicon MicroCantilever, USMC) for hydrogen detection are presented.

\subsection{Resonant frequency shifts of USMC-based sensors}

In recent years, interest in microcantilever-based chemical sensing systems has risen due in part to their projected high sensitivity [3-4]. Because of the large ratio of surface area to volume, the microcantilevers are extremely sensitive to surface processes and can be used as micromechanical sensors [5] for high-resolution sensing and probing [6]. For chemical detection, the USMC-based sensors operated in the dynamic mode are used to measure mass density changes of one scalable analyte in gas mixture [7]. The operating principle of this unconventional use of a microcantilever is based on the fact that the resonant frequency of the microresonator depends on both the density and the viscosity of the surrounding medium. In the case of the out-of-plane mode of a wide microcantilever, it has been shown that the predominant effect causing the change in the microcantilever resonant frequency is due to the gas mass density variation [7]. In fact, when the surrounding fluid mass density increases (decreases), the equivalent effective mass of the microcantilever increases (decreases), thereby causing the resonant frequency to decrease (increase). The absence of a sensitive coating leads to a more reliable and reversible behavior because there is no significant absorption and desorption phenomena. However, the major drawbacks of such sensors is that there is no intrinsic selectivity; thus, these sensors can only be used for specific applications, such as when monitoring environments where it is known that only one gas concentration can vary [8].

The conventional method for monitoring the resonant frequency shift consists of searching for the resonant peak on the amplitude spectrum and tracking the change in the corresponding frequency. The relative variation of the resonant frequency in the case of low concentration of hydrogen $\left(\mathrm{H}_{2}\right)$ in nitrogen $\left(\mathrm{N}_{2}\right)$ is very small. For instance, using an USMC (length=5mm, width=1mm and thickness $=170 \mu \mathrm{m}$, resonant frequency approximately $10 \mathrm{kHz}$ ), a concentration of $0.2 \%$ of $\mathrm{H}_{2}$ in $\mathrm{N}_{2}$ corresponds to a resonant frequency change of approximately $0.02 \mathrm{mHz}$. Therefore, the conventional method does not prove to be sufficiently accurate to measure such a small shift in resonant frequency. This is mainly due to the difficulty of identifying precisely the resonant peak location due to the measurement noise.

The quality factor is a key parameter in resonating sensors because it determines the stability of the resonator in terms of resonant frequency. It is defined as the ratio of the vibrational energy stored in the system to the total energy lost per cycle. This parameter is also commonly used to characterize resonating sensors [9, 10] that are typically used for viscosity measurements [11, 12]. In high quality factor systems, and when the resonant peak is sufficiently high to contain the $-3 \mathrm{~dB}$ bandwidth, the $-3 \mathrm{~dB}$ measurement method is often used to calculate the quality factor. However, when the magnitude of the resonant peak is less than $3 \mathrm{~dB}$, this method cannot be applied.

Other methods for monitoring the shift in resonant frequency consist of making measurements at a fixed frequency instead of using a frequency sweep. By fixing the measurement frequency at the value corresponding to the maximum slope of the gain [13], impedance [14] or phase spectra, changes in the resonant frequency can be detected simply by monitoring variations of response magnitude.

In order to reduce the impact of the noise, polynomial approximations for the gain or phase spectrum can be used. These polynomial approximations can also be used to analytically extract various response characteristics such as natural frequency, resonant frequency and quality factor.

In this paper, the different characterization methods using gain and phase spectra have been tested on measurement data obtained with a USMC device with integrated actuation and read-out systems. To generate measurement data, the sensor has been subjected to various concentrations of hydrogen in nitrogen. The study is focused on the performance of the different characterization 
methods in terms of signal-to-noise ratio (SNR) and the limit of detection (LOD) corresponding to a signal-to-noise ratio of 3. Following a description of the different methods to measure small shifts in resonant characteristics in section 2, the experiments using an uncoated silicon microcantilever-based sensor (USMC) for hydrogen detection are presented in section 3. Finally, a comparison of the results of the different methods applied to these measurements are presented and discussed in section 4.

\section{CHARACTERIZATION METHODS}

In this section, two conventional methods (sections 2.1 and 2.2) and four novel characterization methods (sections 2.3-2.6) are presented.

\subsection{Variation of resonant frequency: $\Delta f_{r}$}

The measurement of the resonant frequency shift, $\Delta f_{r}$, can be simply performed by looking for the resonant peak in the gain spectrum, then associating it with the corresponding frequency as shown in Fig.1.a. However, for small changes of the resonant frequency (USMC case) this technique is not very efficient because of the noise on the gain spectrum inducing significant measurement uncertainty. Nevertheless, eliminating the noise on the gain using appropriate fitting methods (section 2.5) can considerably improve the efficiency of this characterization method.

\subsection{Variation of quality factor: $\Delta Q$}

The conventional measurement method for extracting the quality factor from experimental measurements is the $-3 d B$ bandwidth method, as shown in Fig.1.b. This technique can only be applied to high quality factor systems and it consists of calculating the ratio between the resonant frequency and the $-3 d B$ bandwidth $\left(\Delta f_{-3 d B}\right)$, measured on the gain spectrum:

$$
Q=\frac{f_{r}}{\Delta f_{-3 d B}}
$$

Just as was the case for the $\Delta f_{r}$ method of the previous section, measuring changes in the quality factor by the $-3 d B$ bandwidth method is not effective in the case of small resonant frequency changes and small quality factor changes, because this method requires not only the measurement of $\Delta f_{r}$, but requires also the measurement of $\Delta\left(\Delta f_{-3 d B}\right)$, i.e., the change in the bandwidth.

\subsection{Variation of gain at fixed frequency: $\Delta G_{F F}$}

After setting the measurement (actuation) frequency, $f_{\text {mes }}$, on the point approximately representing the highest slope in the gain spectrum, the characterization of the resonant frequency variation is possible, thanks to the gain variation obtained at $f_{\text {mes }}$ as shown in Fig.1.c.

Moreover, to increase the measurement sensitivity and eliminate the noise on the gain, a linear fit can be applied to the portion of the gain spectrum that includes the maximum slope (inflection point). In practice, it is not efficient to use a general equation to determine the inflection point, because, the shape of the spectrum depends strongly on the coupling between the actuation and sensing circuits (crosstalk). However, a visual inspection of the gain spectrum is sufficient to determine approximately the inflection point, which allows determining the appropriate measurement frequency, $f_{\text {mes }}$, thus improving the efficiency of this characterization method.

Another approach can also be used to eliminate the noise present on the gain spectrum around the resonant peak. In fact, around the resonant frequency, $f_{r}$, the gain spectrum can be approximated by a parabolic curve by using the least-squares method. This approach allows not only the optimization of the $\Delta G_{F F}$ and $\Delta f_{r}$ methods for small-span measurements (span $\leq 4 \mathrm{~Hz}$ in our case), but also the determination of other characteristic parameters as presented in section 2.5.

\subsection{Variation of phase at fixed frequency: $\Delta P_{F F}$}

The linear appearance and high slope around the resonant frequency shown in the phase spectrum (Fig. 1.d) is a major asset for the measurement of small resonant frequency variation, as was seen in the $\Delta G_{F F}$ method presented previously. The $\Delta P_{F F}$ method is 
based on the same principle as the $\Delta G_{F F}$ method; however, in this case the value of $f_{m e s}$ is fixed at the inflection point of the phase spectrum (corresponding to a phase of $-\pi / 2$ ) as shown in Fig.1.d.

The fit of the phase spectrum around the resonant frequency, $f_{r}$, by a straight line, using least-squares method, allows one to not only eliminate noise on the phase and, thus, optimize the performance of this method, but also to deduce other characteristic parameters as discussed in the section 2.6.

\subsection{Parabolic approximation of the gain spectrum near the resonance peak}

As mentioned in the $\Delta f_{r}$ method, to reduce the impact of noise-induced measurement uncertainty on the gain spectrum, a parabolic approximation can be performed around the resonance peak. Using the least-squares method, the $a_{G}, b_{G}$ and $c_{G}$ coefficients in the following fitting equation can be calculated:

$|H| \simeq a_{G}+b_{G} f+c_{G} f^{2}$

The resonant frequency, $f_{r}$, is the maximum of equation (2); thus,

$f_{r}=\frac{-b_{G}}{2 c_{G}}$

The gain transfer function of the microcantilever operating in the linear bending domain is similar to that of a second-order system and is given by

$|H|=\frac{H_{0}}{\sqrt{\left(1-\frac{f^{2}}{f_{0}^{2}}\right)^{2}+\left(2 \xi \frac{f}{f_{0}}\right)^{2}}}$

where $H_{0}$ is the static gain, $f_{0}$ is the natural frequency and $\xi$ the damping ratio.

To determine an analytical parabolic expression of the gain around the resonance peak in terms of system parameters $f_{0}$ and $\xi$, a second-order Taylor series expansion is performed on expression (4), yielding

$|H| \simeq \frac{H_{0}}{2 \xi}\left[3-\frac{1}{2 \xi^{2}}+\left(-3+\frac{1}{\xi^{2}}\right) \frac{f}{f_{0}}+\left(1-\frac{1}{2 \xi^{2}}\right) \frac{f^{2}}{f_{0}^{2}}\right]$

Matching coefficients on equations (2) and (5), we obtain

$$
\left\{\begin{array}{l}
\frac{a_{G} c_{G}}{b_{G}{ }^{2}}=\frac{\left(3-\frac{1}{2 \xi^{2}}\right)\left(1-\frac{1}{2 \xi^{2}}\right)}{\left(-3+\frac{1}{\xi^{2}}\right)^{2}} \\
H_{0}=\frac{2 a_{G} \xi}{3-\frac{1}{2 \xi^{2}}} \\
\frac{a_{G}}{b_{G}}=f_{0} \frac{3-\frac{1}{2 \xi^{2}}}{-3+\frac{1}{\xi^{2}}}
\end{array}\right.
$$

Equation 6.a may be rewritten to give an explicit expression for the quality factor in terms of the fitting coefficients: 
$Q=\frac{1}{2 \xi}=\sqrt{\frac{(3 A-1)-\frac{1}{2} \sqrt{1-3 A}}{4 A-1}}$

with $A=\frac{a_{G} c_{G}}{b_{G}{ }^{2}}$.

\section{Remarks:}

- The parabolic approximation of the gain is only valid on the part of the gain spectrum very close to the resonance (frequency $\left.\operatorname{span}<\Delta f_{-3 d B}\right)$.

- The second root, $\frac{1}{2 \xi}=\sqrt{\frac{(3 A-1)+\frac{1}{2} \sqrt{1-3 A}}{4 A-1}}$, of equation (6a) gives a false $Q$-factor value, so only the root given by equation (7) is considered.

\subsection{Linear approximation of the phase spectrum near the resonance peak}

In the linear bending regime the phase transfer function of the microcantilever can be written as

$$
\varphi(f)=-\operatorname{Arctan}\left(\frac{2 \xi \frac{f}{f_{0}}}{1-\frac{f^{2}}{f_{0}^{2}}}\right)
$$

A linear fit of the phase spectrum around $f_{0}$ using the least-squares method allows for the numerical determination of the coefficients $a_{P}$ and $b_{P}$ in the fitting equation below:

$\varphi(f)=a_{P}+b_{P} f$

(9)

Furthermore, an analytical expression of the phase in the vicinity of the eigenfrequency $f_{0}$ may be used to determine $f_{0}$ and $\xi$ of the microcantilever. To obtain this expression, a first-order Taylor series expansion is performed on the phase transfer function around $f_{0}$ (i.e., where the phase is to $-\pi / 2$ ). The result of this calculation yields

$\varphi(f)=-\frac{\pi}{2}+\frac{1}{\xi}\left(1-\frac{f}{f_{0}}\right)$

from which one may obtain the following:

$$
\left\{\begin{array}{l}
Q=\frac{1}{2 \xi}=-\frac{1}{2}\left(\frac{\pi}{2}+a_{P}\right) \\
f_{0}=\frac{1}{\xi b_{P}}=\frac{-\frac{\pi}{2}-a_{P}}{b_{P}}
\end{array}\right.
$$

\section{Remarks:}

- The linear approximation of the phase is only valid in the part of the phase spectrum that is very close to resonance (span $<\Delta f$. $3 d B)$. 
- Once $f_{0}$ and $\xi$ are calculated, it is easy to deduce the resonant frequency: $f_{r}=f_{0} \sqrt{1-2 \xi^{2}}$
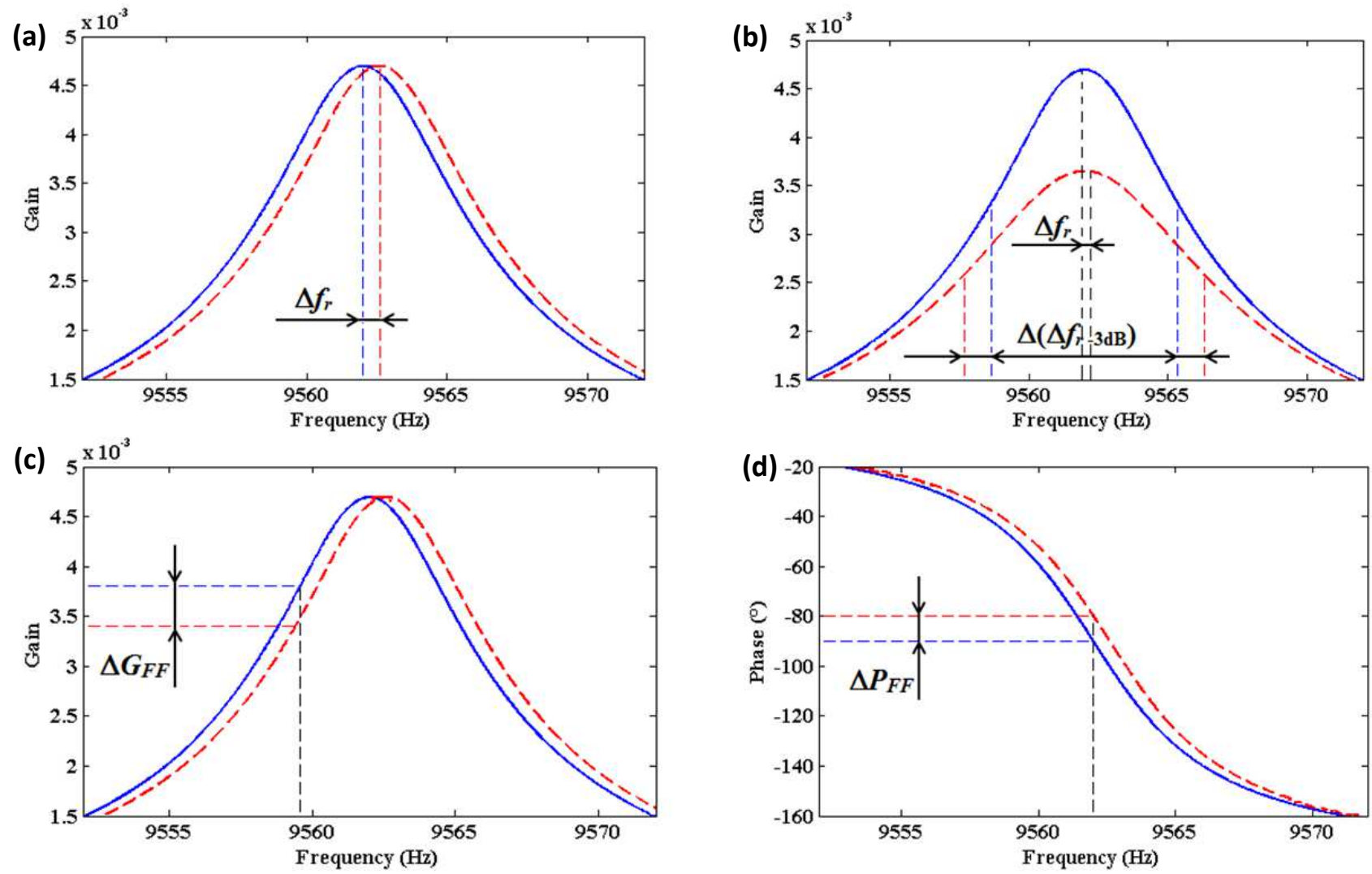

Fig. 1 : Principle of the different characterization methods: (a) $\Delta f_{r}$ : measurement of the resonant frequency variation corresponding to the maximum of the gain. (b) $\Delta Q$ : measurement of the quality factor variation using the $-3 \mathrm{~dB}$ bandwidth method, (c) $\Delta G_{F F}$ : measurement of the gain variation at a fixed frequency, $f_{m e s}$, corresponding approximately to the maximum slope of the gain, $(\mathrm{d}) \Delta \boldsymbol{P}_{F F}$ : measurement of the phase variation at a fixed frequency, $f_{\text {mes }}$, corresponding approximately to the maximum slope of the phase.

\section{EXPERIMENTS}

In order to apply the different characterization methods presented in the section 2, measurements of hydrogen in nitrogen have been conducted using: (1) a USMC with integrated electromagnetic actuation and piezoresistive sensing (Fig.2), and (2) a gas line for mixing gas with controlled flow and concentrations (Fig.3).

\subsection{Microcantilever design}

The microcantilever has been fabricated by ESIEE group (Paris, France, www.esiee.fr) using classical silicon technologies [15].

To generate vibrations, an AC current is passed through the conductive wire placed at the cantilever periphery. In the presence of a magnetic field collinear to the longitudinal axis of the beam, an AC Lorenz force is created at the microcantilever free-end and induces out-of-plane vibrations. In order to detect these vibrations, semiconductor strain gauges (boron-doped piezoresistors) are fabricated during the process. They are arranged in a half Wheatstone bridge configuration: a first gauge is located where the strains are maximum (at the clamped-end of the beam) and the other one is on the rigid substrate (Fig.2). The USMC characteristics in air at $23 \mathrm{C}^{\circ}$ are: length $=5 \mathrm{~mm}$, width $=1 \mathrm{~mm}$, thickness $=170 \mu \mathrm{m}, f_{r} \approx 9662 \mathrm{~Hz}$ and $Q \approx 1350$. 


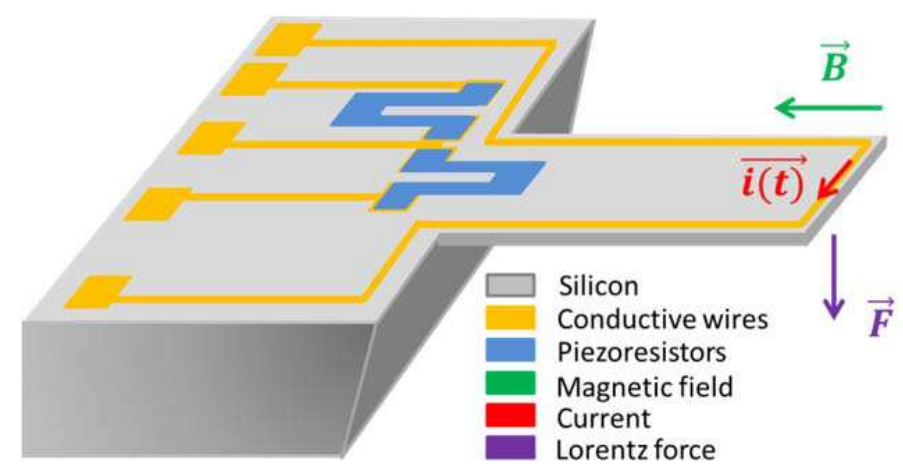

Fig. 2 : Uncoated silicon microcantilever-based sensor (USMC) design: a conductive wire is placed at the free-end of the cantilever for the electromagnetic actuation by Lorentz force; piezoresistors are placed on the clamped-end of the cantilever and on the substrat for cantilever vibration measurement.

\subsection{Experimental setup}

The microcantilever is placed in a hermetic gas chamber (total volume: $500 \mu \mathrm{l}$ ) under a controlled gas flow. The gas line (Fig.3) contains mass-flowmeters allowing control of flow (100-1000 $\mathrm{ml} / \mathrm{min})$ and concentration of each gas. To perform chemical detection, a gas stream containing a $\mathrm{H}_{2}-\mathrm{N}_{2}$ mixture flows into the gas chamber in which the USMC has been placed. The tested succession of the different concentrations of $\mathrm{H}_{2}$ in $\mathrm{N}_{2}$ is reported in different detection curves.



Fig. 3 : Experimental setup. Gas line constituted of hydrogen $\left(4 \% \mathrm{H}_{2}-\mathrm{N}_{2}\right)$ and nitrogen $\left(100 \% \mathrm{~N}_{2}\right)$ gas bottles and mass-flowmeters controlled by a computer. The uncoated silicon microcantilever-based sensor is put in a hermetic gas chamber connected to the $\mathrm{H}_{2}-\mathrm{N}_{2}$ mixture gas flow (usually $100 \mathrm{ml} / \mathrm{min}$ but can be increased up to $1000 \mathrm{ml} / \mathrm{min}$ ).

In order to study the behavior of the USMC in the presence of different concentrations of $\mathrm{H}_{2}$ in $\mathrm{N}_{2}$, a spectrum analyzer (HP4194A) is used to acquire gain and phase spectra with the following configuration: INTEG.TIME=Short, AVRG=8bits. The feature of this device is that it has only 400 measurement points. Therefore, for small $f_{r}$ shifts $(\leq 1 \mathrm{~Hz})$, small spans must be applied to increase the measurement sensitivity. For example, to detect $f_{r}$ variations of at least $20 \mathrm{mHz}$, a measurement span of no more than $8 \mathrm{~Hz}(8 \mathrm{~Hz} / 400$ points $)$ can be used. In our case a span of $4 \mathrm{~Hz}$ is used for the detection of different $\mathrm{H}_{2}$ in $\mathrm{N}_{2}$ concentrations. Each $\mathrm{H}_{2}$ in $\mathrm{N}_{2}$ concentration lasts 3 minutes with a constant flow of $100 \mathrm{ml} / \mathrm{min}$.

After following the experimental protocol detailed previously, the gain and phase spectra obtained for the concentrations of $\mathrm{H}_{2}$ in $\mathrm{N}_{2}$ are presented in Fig.4. The gain and phase translations to the right observed in Fig.4.a and Fig.4.b, respectively, are consistent with the decrease in the gas mass density when the $\mathrm{H}_{2}$ concentration increases. This type of resonant frequency shift in the USMC due to density changes in the surrounding gas has been discussed in an earlier work [7]. 

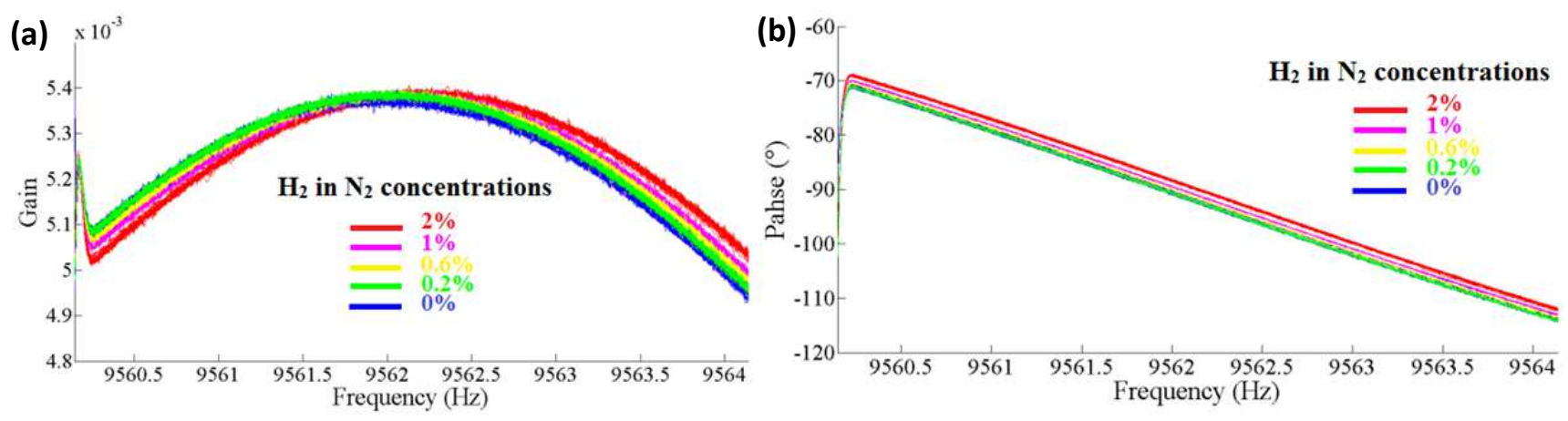

Fig. 4 : Evolution of spectra of the USMC $(5 \mathrm{~mm} \times 1 \mathrm{~mm} \times 170 \mu \mathrm{m})$ near the resonance as $\mathrm{H}_{2}$ in $\mathrm{N}_{2}$ concentration is changed from $0 \%$ to $2 \%$ with a gas flow of $100 \mathrm{ml} / \mathrm{min}$. (a) Gain spectrum. (b) Phase spectrum. Small modifications of both the gain and phase spectra are observed due to the increase of the resonant frequency with the hydrogen concentration.

\section{RESULTS AND DISCUSSION}

In this section, the different characterization methods presented in this paper are tested. In order to compare performances of these methods in terms of signal-to-noise ratio, SNR, the relative variation of $f_{r}, f_{0}, Q, G_{F F}$ and $P_{F F}$ are presented.

The results of Fig.5 demonstrate the validity of polynomial approximations of gain and phase spectra around resonance for a frequency span of $4 \mathrm{~Hz}$. These approximations are used to fit spectra, enabling one to increase the SNR (section 4.1) and to deduce $f_{r}, f_{0}$ and $Q$ (section 4.2) using equations (3), (7) and (11). The measurement frequencies $\left(f_{\text {mes }}\right.$ ), which are different for the $\Delta G_{F \mathrm{~F}}$ method and $\Delta P_{F F}$ method, are also indicated in the gain (Fig.5.a) and phase (Fig.5.b) spectra; they are respectively placed at the maximum slope of the gain $\left(f_{m e s}=9564 \mathrm{~Hz}\right)$ for the $\Delta G_{F F}$ method and at the natural frequency $\left(f_{\text {mes }}=f_{0}=9562 \mathrm{~Hz}\right)$ for the $\Delta P_{F F}$ method.
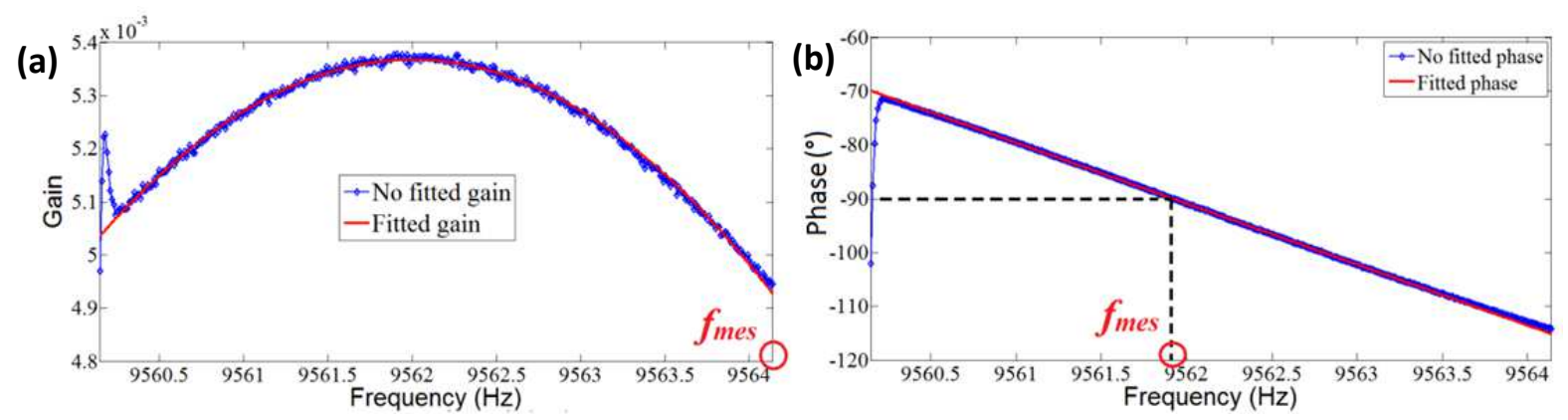

Fig. 5 : Agreement between least-squares polynomial approximations and raw spectra. (a) Gain spectrum. (b) Phase spectrum. The measurement frequencies are shown for both the gain measurement (highest frequency of the measurements which corresponds to the maximum slope of the gain) and the phase measurement (frequency corresponding to a phase equal to $-90^{\circ}$ which corresponds to the maximum slope of the phase).

\subsection{Performance of the $\Delta f_{r}, \Delta G_{F F}$ and $\Delta P_{F F}$ methods}

The detection results of the $\Delta f_{r}, \Delta G_{F F}$ and $\Delta P_{F F}$ characterization methods, obtained for concentrations of $\mathrm{H}_{2}$ in $\mathrm{N}_{2}$ ranging from $2 \%$ to $0.2 \%$ are presented in Fig. 6 .

As expected, monitoring $f_{r}$ by the conventional method without fitting the gain spectrum is not efficient because of the noise on the gain (Fig.6.a). However, fitting the gain with a parabolic approximation and using relation (3) to monitor $f_{r}$ drastically improves the SNR (blue line in Fig.6.a). 
The $\Delta G_{F F}$ (Fig. 6.b) and $\Delta P_{F F}$ (Fig.6.c) methods show good sensitivity; they are able to detect a concentration of $0.2 \%$ of $\mathrm{H}_{2}$ in $\mathrm{N}_{2}$ even without fitting. Moreover, the polynomial fits have greatly improved the SNR of both methods (blue lines). Table 1 presents the performance of each method, calculated at $0.6 \%$ of $\mathrm{H}_{2}$ in $\mathrm{N}_{2}$. The noise is estimated by calculating the standard deviation $\left(\sigma_{\mathrm{x}}\right)$. From Table 1, it can be seen that the $\Delta P_{F F}$ method has the best $\mathrm{SNR}(\mathrm{S} / \mathrm{N}=100)$, the best relative variation $(\Delta x / x=0.86 \%)$ and the best limit of detection (hydrogen concentration corresponding to a SNR equal to $3,=0.018 \%$ of $\mathrm{H}_{2}$ in $\mathrm{N}_{2}$ ).

The performance of the $\Delta G_{F F}$ method can be optimized by fixing $f_{\text {mes }}$ at the point of maximum slope of the gain spectrum. Thus, another experiment is carried in the same conditions (the same concentrations of $\mathrm{H}_{2}$ in $\mathrm{N}_{2}$ and the performances are calculated at $0.6 \%$ of $\mathrm{H}_{2}$ in $\mathrm{N}_{2}$ ), but using a frequency span of $10 \mathrm{~Hz}$ in order to identify the maximum slopes of both the gain and phase spectra. In these conditions, the parabolic fitting cannot be applied to the whole spectrum (Fig.7, red line with ' $x$ ' marks); it can only be applied closely around resonance (Fig.7, green line with triangle marks). However, for large span measurements only the tangent to the curve at the inflection point (Fig.7, black line with circle marks) is used to improve the SNR. The performance of each method is reported in Table 2. The comparison between the $\Delta G_{F F}$ and $\Delta P_{F F}$ methods in their optimal conditions shows that the $\Delta P_{F F}$ method yields a SNR of 32 in comparison with 25 for the $\Delta G_{F F}$ method, thereby confirming that $\Delta P_{F F}$ is the best characterization method that we have examined to date. This is due to the fact that the phase spectrum presents a higher slope and a lower noise than the gain spectrum.

\section{Remark:}

Because of the large frequency span (10Hz) chosen in this measurement, the signal-to-noise ratios (Table 2) are relatively small compared to the values found in Table 1. This is due to the configuration of the gain/phase analyzer (HP5194A) that is maintained at the same level (INTEG.TIME=Short, AVRG=8bits) as for the measurements performed with the short span (4Hz). This results in a higher noise level associated with the larger frequency span.
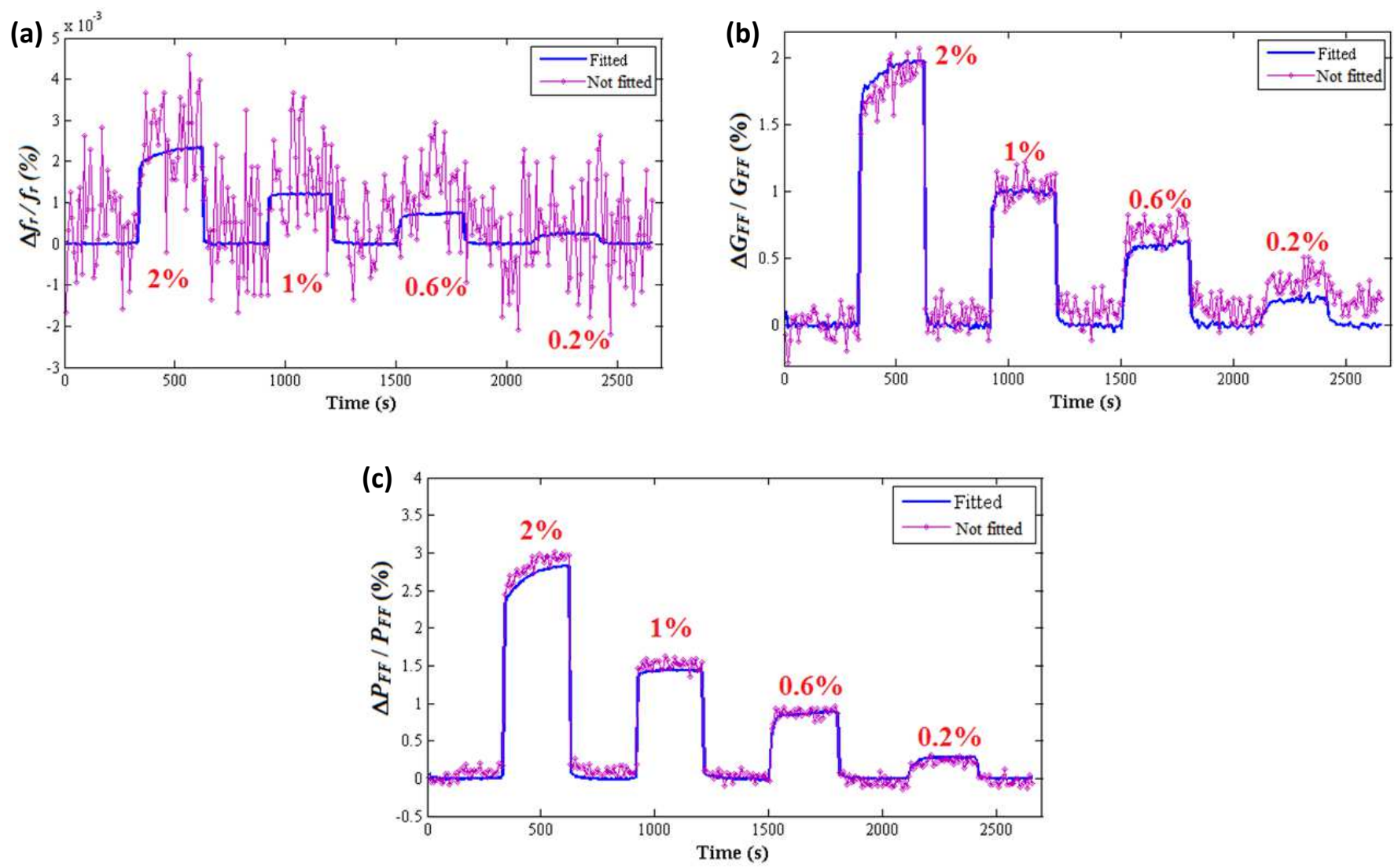

Fig. 6 : Detection curves of the USMC $(5 \mathrm{~mm} \times 1 \mathrm{~mm} \times 170 \mu \mathrm{m})$ as $\mathrm{H}_{2}$ in $\mathrm{N}_{2}$ concentration is changed from $2 \%$ to $0.2 \%$ with a gas flow of $100 \mathrm{ml} / \mathrm{min}$. The duration of each concentration step is $3 \mathrm{~min}$ and between each concentration step pure nitrogen is injected in the gas chamber during $3 \mathrm{~min}$. The same measurements are used to test the three different methods: (a) $\Delta f_{r}$ method. (b) $\Delta G_{F F}$ method. (c) $\Delta P_{F F}$ method. 


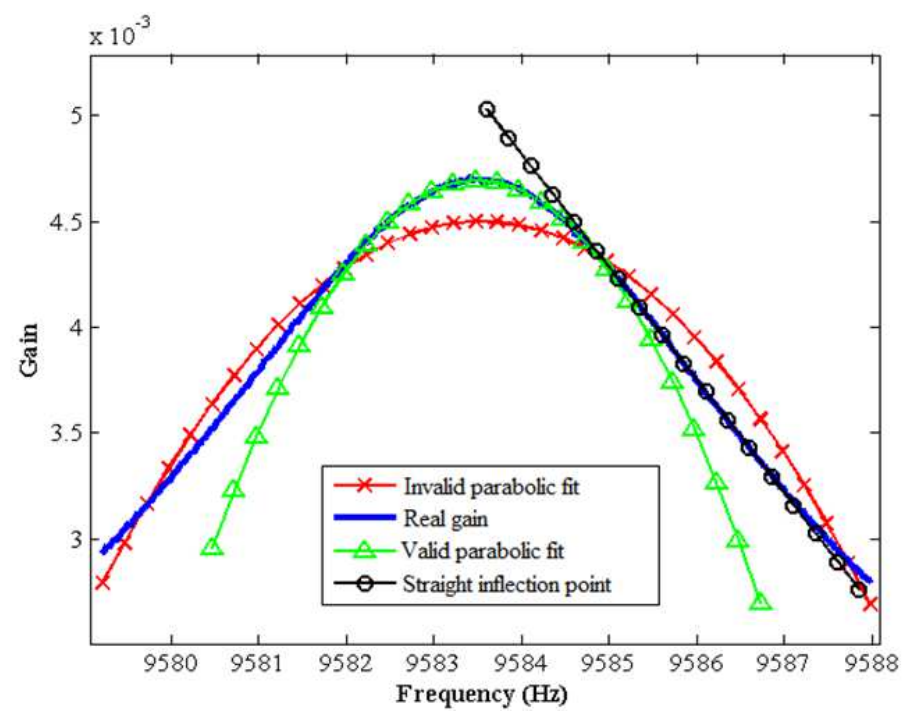

Fig. 7 : Comparison between the real gain spectrum and the various fits adopted for the USMC (5mm x $1 \mathrm{~mm} \times 170 \mu \mathrm{m})$ with a $10 \mathrm{~Hz}$ span around the resonant frequency.

Table 1 : Performance of $\Delta P_{F F}$ and $\Delta f_{r}$ methods $\left(\Delta x / x\right.$ : relative variation. $\sigma_{x}$ : noise estimation (standard deviation of the measured relative variation). LOD: limit of detection). Frequency span $=4 \mathrm{~Hz}$.

Characterization methods

$$
\left(\frac{\Delta x}{x}\right)_{0.6 \% H_{2}}(\%) \quad \sigma_{x}(\%) \quad\left(\frac{S}{N}\right)_{0.6 \% H_{2}}
$$

\begin{tabular}{|c|c|c|c|c|c|}
\hline \multirow{3}{*}{$\begin{array}{l}\text { Without } \\
\text { fitting }\end{array}$} & $\Delta f_{r}$ & - ------ & $0.82 \times 10^{-3}$ & ----- & ------- \\
\hline & $\Delta G_{F F}$ & 0.72 & $82 \times 10^{-3}$ & 8.8 & $0.2 \%$ \\
\hline & $\Delta P_{F F}$ & 0.88 & $54 \times 10^{-3}$ & 16 & $0.11 \%$ \\
\hline \multirow{3}{*}{$\begin{array}{l}\text { With } \\
\text { fitting }\end{array}$} & $\Delta f_{r}$ & $0.72 \times 10^{-3}$ & $15 \times 10^{-6}$ & 48 & $0.037 \%$ \\
\hline & $\Delta G_{F F}$ & 0.60 & $16 \times 10^{-3}$ & 38 & $0.047 \%$ \\
\hline & $\Delta \boldsymbol{P}_{F F}$ & 0.86 & $8.5 \times 10^{-3}$ & 100 & $0.018 \%$ \\
\hline
\end{tabular}

Table 2 : Performance of $\Delta P_{F F}$ and $\Delta G_{F F}$ methods ( $\Delta x / x$ : relative variation. $\sigma_{x}:$ noise estimation (standard deviation of the measured relative variation). LOD: limit of detection). Frequency span $=10 \mathrm{~Hz}$.

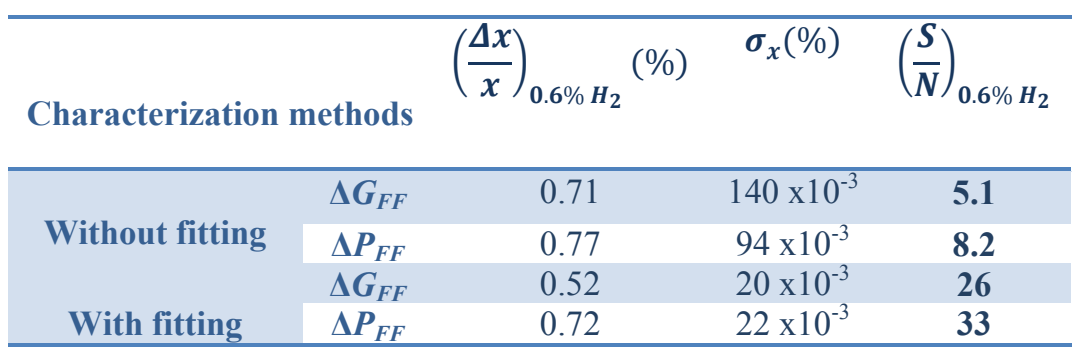

\subsection{Performance of polynomial approximation methods}


In the previous part, polynomial approximations using the least-squares method were applied to fit the gain and phase spectra near resonance in order to decrease the impact of the noise. Using the polynomial coefficients of the fitting methods, equations (3), (7), (6.c) and (11) can be used to deduce $f_{r}, Q$ and $f_{0}$. In this experiment, we compare only the performance of each fitting method in terms of the determination of $f_{0}$ and $Q$. The results of this processing and the corresponding performance values are presented in Fig. 8 and Table 3, respectively, for a parabolic approximation of the gain and a linear approximation of the phase.

It can be observed that variations of $Q$ and $f_{0}$ in the presence of different concentrations of $\mathrm{H}_{2}$ in $\mathrm{N}_{2}$ are detected using the different polynomial methods. All performance metrics are reported in Table 3, where it can be seen that $Q$ measurements (green lines with stars in Fig. 8) present a poor SNR (2.5 and 1.8 for gain and phase fits, respectively) and cannot detect $\mathrm{H}_{2}$ concentrations in $\mathrm{N}_{2}$ below $1 \%$. Also, the actual $Q$ value is around 1350 , while the measured $Q$ values based on the polynomial approximations are approximately 900-950. This estimation error is mostly caused by the span measurement $(4 \mathrm{~Hz})$ which is relatively large in comparison with the range of validity of these polynomial methods. However, the exact $Q$ measurement is not necessary; only the $Q$ variation is important in the present application, i.e., to sufficiently characterize USMC response to a change in $\mathrm{H}_{2}$ concentration in $\mathrm{N}_{2}$. On the other hand, $f_{0}$ measurements utilizing both the gain and phase polynomial methods (blue lines in Fig. 8) yield a good SNR (46 and 82 for gain and phase spectra, respectively) and a good $f_{0}$ estimation (at $0 \%$ of $\mathrm{H}_{2}$ in $\mathrm{N}_{2}$, temperature $\approx 23 \mathrm{C}^{\circ}$ and $f_{0} \approx$ $9562 \mathrm{~Hz}$ ). The comparison between the $f_{0}$ determinations shows that the linear approximation of the phase near resonance results in the best SNR and consequently the best LOD. 


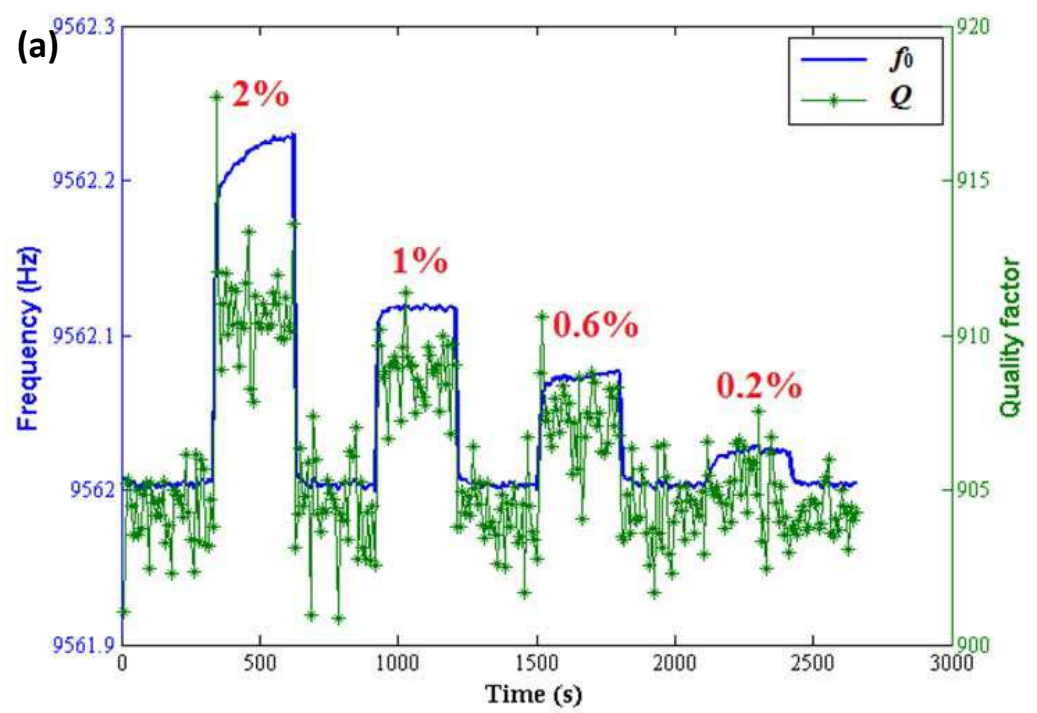

(b)

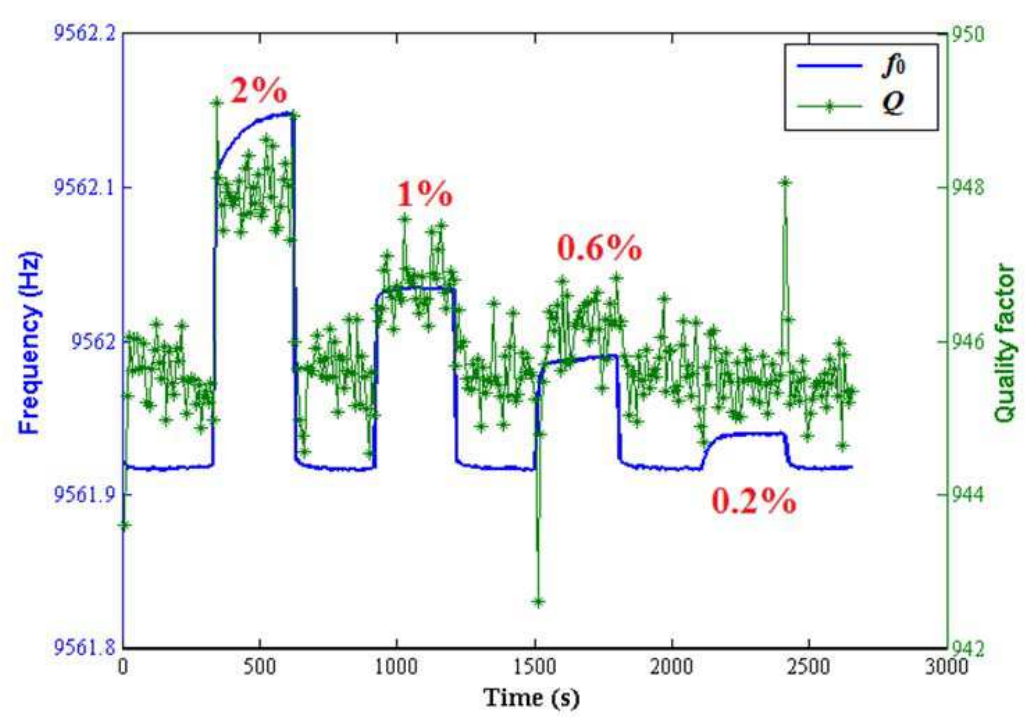

Fig. 8 : Detection curves of the USMC $(5 \mathrm{~mm} \times 1 \mathrm{~mm} \times 170 \mu \mathrm{m})$ as $\mathrm{H}_{2}$ in $\mathrm{N}_{2}$ concentration is changed from $2 \%$ to $0.2 \%$ with a gas flow of $100 \mathrm{ml} / \mathrm{min}$. The duration of each concentration step is $3 \mathrm{~min}$ and between each concentration step pure nitrogen is injected in the gas chamber during $3 \mathrm{~min}$. It is the same data measurements as the one of Fig 6 and the methods presented in sections 2.5 and 2.6 are tested.

(a) The parabolic approximation of the gain spectrum near the resonance peak is used and both the eigenfrequency $f_{0}$ and the quality factor $Q$ are plotted using Eqs. 6 c and 7; (b) the linear approximation of the phase spectrum near the resonance peak is used and both the aigenfrequency $f_{0}$ and the quality factor $Q$ are plotted using Eqs. 11a and 11b.

Table 3 : Performance of polynomial gain and phase approximations ( $\Delta x / x$ : relative variation. $\sigma_{x}$ : noise estimation (standard deviation of the measured relative variation). LOD: limit of detection). Frequency $\operatorname{span}=4 \mathrm{~Hz}$.

\begin{tabular}{|c|c|c|c|c|c|}
\hline \multicolumn{2}{|l|}{ Characterization methods } & $\left.\frac{\Delta x}{x}\right)_{0.6 \% H_{2}}(\%)$ & $\sigma_{x}(\%)$ & $\left(\frac{S}{N}\right)_{0.6 \% H_{2}}$ & LOD \\
\hline \multirow[t]{2}{*}{ Parabolic approximation: gain fitting } & $\Delta Q$ & $330 \times 10^{-3}$ & $130 \times 10^{-3}$ & 2.5 & $0.72 \%$ \\
\hline & $\Delta f_{0}$ & $0.73 \times 10^{-3}$ & $16 \times 10^{-6}$ & 46 & $0.04 \%$ \\
\hline \multirow[t]{2}{*}{ Linear approximation : phase fitting } & $\Delta Q$ & $74 \times 10^{-3}$ & $41 \times 10^{-3}$ & 1.8 & $1 \%$ \\
\hline & $\Delta f_{0}$ & $0.74 \times 10^{-3}$ & $9 \times 10^{-6}$ & 82 & $0.022 \%$ \\
\hline
\end{tabular}

\subsection{Limit of detection}


The analysis of the performance tables (Tables 1-3) shows that phase linearization around resonance optimizes the measurement sensitivity of the resonant frequency variation using the $\Delta P_{F F}$ method ( $\left.\mathrm{SNR}=100\right)$. Therefore, it would be interesting to assess the corresponding limit of detection (LOD).

In theory, the LOD of the $\Delta P_{F F}$ method is approximately $0.02 \%$ of $\mathrm{H}_{2}$ in $\mathrm{N}_{2}$. To verify this value, another experiment is carried out using a concentration of $0.025 \%$ of $\mathrm{H}_{2}$ in $\mathrm{N}_{2}$. Because of the accuracy limit of the flowmeters, the gas flow is increased in this experiment to $200 \mathrm{ml} / \mathrm{min}$ in order to achieve this low concentration of $\mathrm{H}_{2}$ in $\mathrm{N}_{2}$. The measurement result using the $\Delta P_{F F}$ method is presented in Fig. 9, where it can be seen that a concentration of $200 \mathrm{ppm}(0.025 \%)$ has been successfully detected.



Fig. 9 : Detection curve of the USMC $(5 \mathrm{~mm} \times 1 \mathrm{~mm} \times 170 \mu \mathrm{m})$ using the $\Delta P_{F F}$ method as $\mathrm{H}_{2}$ in $\mathrm{N}_{2}$ concentration is changed from $0 \%$ to $0.025 \%$ and to $0 \%$ with a gas flow of $200 \mathrm{ml} / \mathrm{min}$..

In summary, the phase linearization using the least-squares method and the linear model around resonance to measure the phase variation at a fixed frequency (Table 1) or the natural frequency variation (Table 3) present the best SNRs among all characterization methods studied in this work. This is due to the fact that the phase spectrum results in lower noise and a higher slope compared to the gain spectrum. Using the $\Delta P_{F F}$ method, $0.025 \%$ of $\mathrm{H}_{2}$ in $\mathrm{N}_{2}$ has been detected using an USMC with the characteristics mentioned in section 3.1 .

Unlike the conventional resonant frequency variation method $\left(\Delta f_{r}\right)$, the $\Delta P_{F F}$ method allows accurate measurement of small resonant frequency variation of the USMC, thanks to its high phase slope around resonance (high $Q$ ). In addition, the linearized phase model enables one to extract an accurate value of natural frequency variation $\left(\Delta f_{0}\right)$ and to monitor accurately both small and large (if the frequency span is large enough) resonant frequency variations (but with less sensitivity than the $\Delta P_{F F}$ method for small resonant frequency variation).

Another advantage of the $\Delta P_{F F}$ method is the simplicity involved in making an integrated measurement with only one circuit that performs the phase comparison between the actuation signal at a fixed frequency $\left(f \approx f_{r}\right)$ and the read-out signal. The major drawback of this method is that it can only be used for small resonant frequency variation (due to the smallness of phase linearity domain).

\section{Acknowledgement}

This work is supported by Andra. The authors are grateful for interesting discussions with Stephen Heinrich on the paper content and for his suggestions for improving the paper. 


\section{References}

[1] S. Buschaert, S. Lesoille-Delepine, J. Bertrand, S. Mayer, P. Landais, Developing the tools for geologic repository monitoring - Andra's monitoring R\&D program, Proc. of the WM2012 conference, Phoenix, 2012.

[2] T. Hübert, L. Boon-Brett, G. Black, U. Banach, Hydrogen Sensors- A review, Sensors and Actuators B, 157 (2011), $329-352$.

[3] K.M. Goeders, J.S. Colton, L. A. Bottomley, Microcantilevers: sensing chemical interactions via mechanical motion, Chemical Reviews, 108 (2008), 522-542.

[4] Q. Zhu, Microcantilever sensors in biological and chemical detections, Sensors \& Transducers Journal, 125 (2011), 1-21.

[5] A. Boisen, S. Dohn, S.S. Keller, S. Schmid, M. Tenje, Cantilever-like micromechanical sensors, Reports on Progress in Physics, 74 (2011), 036101.

[6] D. W. Lee, X. Li, Integrated microcantilevers for high-resolution sensing and probing, Measurement Science and Technology 23 (2012), 029501.

[7] S. Tétin , B. Caillard, F. Ménil, H. Debéda, C. Lucat, C. Pellet, I. Dufour, Modeling and performance of uncoated microcantilever-based chemical sensors, Sensors and Actuators B, 143 (2010), 555-560.

[8] I. Dufour, F. Josse, S. Heinrich, C. Lucat, C. Ayela, F. Ménil, O. Brand, Unconventional uses of microcantilevers as chemical sensors in gas and liquid media, Sensors and Actuators B, 170 (2012), 115-121.

[9] S. Schmid, P. Senn, C. Hierold, Electrostatically actuated nonconductive polymer microresonators in gaseous and aqueous environment, Sensors and Actuators A, 145-146 (2008), 442-448.

[10] J.E.Y. Lee, Y. Xu, Direct inference of parameters for piezoresistive micromechanical resonators embedded in feedthrough, Sensors and Actuators A, 186 (2012), 257-263.

[11] A. Abdallah, M. Heinisch, B. Jakoby. Measurement error estimation and quality factor improvement of an electrodynamicacoustic resonator sensor for viscosity measurement, Sensors and Actuators A, 199 (2013), 318-324.

[12] A.M. Schilowitz, D.G. Yablona, E. Lansey, F.R. Zypman, Measuring hydrocarbon viscosity with oscillating microcantilevers, Measurement, 41 (2008), 1169-1175.

[13] T.R Albrecht, P. Grütter, D. Horne, D. Rugar, Frequency modulation detection using high-Q cantilevers for enhanced force microscope sensitivity, Journal of Applied Physics, 69 (1991), 668-673.

[14] S. Xu, R. Mutharasan, A novel method for monitoring mass-change response of piezoelectric-excited millimeter-sized cantilever (PEMC) sensors, Sensors and Actuators B, 143 (2009), 144-151.

[15] L. Fadel, F. Lochon, I. Dufour, O. Français, Chemical sensing: millimeter size resonant microcantilever performance, Journal of Micromechanics and Microengineering, 14 (2004), S23-S30.

\section{Biographies}

Mohand-Tayeb Boudjiet received in 2012 the Master degree in embedded electronics systems from University of Bordeaux in France. He is currently a Ph.D student at IMS Laboratory and he is working on microcantilevers for density measurement and chemical detections.

Johan Bertrand received in 2005 the M.S. degree solid and inorganic chemistry from Rennes 1 University, France. In 2009, he got a Ph.D degree in physic and chemistry from the University of Tubingen and École des Mines de Saint-Étienne. Since 2009, he works for the National Radioactive Waste Management Agency as a Research Engineer working an overall monitoring strategy and sensors development for the deep geological disposal for high-level and long-lived radioactive waste.

Claude Pellet was a researcher at the "Institut d'Electronique Fondamentale" from the university Paris XI-Orsay, where he has studied the deposition of thin film by ion beam sputtering from 1982 to 1993 . He joined university of Bordeaux as full professor in 1993. His work focuses on micro -technology, micro-system development (humidity sensor), assembly technology and microsystems reliability. He is currently the director of the IMS Laboratory (400 people).

Isabelle Dufour graduated from Ecole Normale Supérieure de Cachan in 1990 and received the Ph.D. and H.D.R. degrees in engineering science from the University of Paris-Sud, Orsay, France, in 1993 and 2000, respectively. She was a CNRS researchfellow from 1994 to 2007, first in Cachan working on the modelling of electrostatic actuators (micromotors, micropumps) and then after 2000 in Bordeaux working on microcantilever-based chemical sensors. She is currently Professor of electrical 
Post-print: Sensors and Actuators B, 199, 2014, pp. 269-276 (doi: 10.1016/j.snb.2014.03.098)

engineering at the University of Bordeaux and her research interests are in the areas of microcantilever-based sensors for chemical detection, rheological measurements, material characterization and energy harvesting. 